\title{
DIE FAMILIE V. LIPHART IM SPIEGEL IHRES ARCHIVS IM HERDER-INSTITUT MARBURG
}

\author{
EINLEITUNG
}

Die Familie von Liphart muss in baltischen wie auch in kultur- und kunsthistorischen Kreisen kaum mehr vorgestellt werden. Der Name Liphart steht für: „fünf Generationen bedeutende Kunstsammler"1, und das bedeutende Gutshaus der Familie v. Liphart, Schloss Ratshof bei Dorpat, wird allgemein der „Musenhof ${ }^{\prime 2}$ genannt. In vielerlei Äußerungen wird die Familie mit den Begriffen Kunstsammler und Mäzene und als Förderer und Unterstützer der Kunst und von Künstlern und ganz allgemein mit der Kultur im Baltikum verbunden. ${ }^{3}$

Mit der Familiengeschichte, insbesondere aber mit den agrargeschichtlichen Wirkungen der verschiedenen Familienzweige Liphart hat sich Tiit Rosenberg, Dorpat/Tartu, eingehend beschäftigt ${ }^{4}$; wie wichtig die erneute Beschäftigung mit dieser Familie und besonders der

DOI: http://dx.doi.org/10.12697/BJAH.2016.12.04

Die Verf.in greift hier Ergebnisse einer früheren Studie über die Familie v. Liphart auf: Dorothee M. Goeze: „,Von der Pflicht, anregend, fördernd auf das geistige Leben seiner Mitbürger einzuwirken.” Die Familie von Liphart im Spiegel ihres Archivs im Herder-Institut Marburg", Jahrbuch des baltischen Deutschtums, Bd. 56 (2009), hrsg. von der Carl-Schirren-Gesellschaft e. V. (Lüneburg, 2008), 72-82.

1 Vgl. Deutschbaltisches Biographisches Lexikon 1710-1960, hrsg. von Wilhelm Lenz (Köln: Böhlau Verlag, 1970), 463.

2 Vgl. den Untertitel des Bandes: Erik Thomson, Georg von Rauch, Schloß Ratshof in Estland: vom Musenhof zum Nationalmuseum (Lüneburg: Nordostdeutsches Kulturwerk, 1985).

3 Vgl. exemplarisch: Juta Keevallik, „Karl Eduard von Liphart”, Jahrbuch des baltischen Deutschtums, 39 (1992), 38-41, dazu noch: Unistuste Raadi. Liphartite kunstikogu Eestis = The Liphart family and their art collection in Estonia (Tartu: Tartu Ülikooli Kirjastus, 2015).

4 Tiit Rosenberg, „Liphartid Liivimaa mõisnikuna I = Die Lipharts als Gutsbesitzer Livlands”, Kleio, 9 (1994), 12-16; ders.: „Liphartid Liivimaa mõisnikuna II”, Kleio, 2 (16) (1996), 19-23; ders: „Liphartid Liivimaa mõisnikuna III”, Kleio, 3 (21) (1997), 8-17. 
in Jahrhunderten zusammengetragenen Kunstsammlungen, ist, zeigt ein in Estland Forschungs-Projekt zum Thema Kunstsammlungen der Familie v. Liphart. ${ }^{5}$

Vorliegende Ausführungen sollen Anregung sein, sich mit dem Archivmaterial zu beschäftigen (so wie es die Bearbeiter des vorliegenden Bandes getan haben) und damit die Familie v. Liphart und ihre Geschichte, die inneren Zusammenhänge einer bedeutenden Adelsfamilie und das Wirken einzelner Personen als Gönner und Mäzene in der Welt der Wissenschaften und der Künste Livlands darzustellen.

\section{DAS FAMILIENARCHIV V. LIPHART IN DER DOKUMENTESAMMLUNG DES HERDER-INSTITUTS} MARBURG (DSHI)

Der Bestand „Familienarchiv Liphart” in der Dokumentesammlung des Herder-Instituts besteht aus zwei Teilen, die auf Wunsch der jeweiligen unterschiedlichen Eigentümer physisch getrennt aufbewahrt werden, inhaltlich aber natürlich eng zusammengehören.

Nach der Enteignung von Ratshof 1920 und nach der Übersiedlung des letzten Majoratsherrn nach Deutschland wurden viele Dinge (Gemälde, Bücher, persönliche Papiere, Akten u. a.) zerstreut, vieles ging verloren, vieles wurde im Zweiten Weltkrieg in Deutschland durch Kriegseinwirkung zerstört. Einige Teile gelangten jedoch nach 1945 in die USA. Von dort aus wurde durch den Erben, Herrn Laurence Bingenheimer-Emich, im Jahr 2000 das ihm verfügbare Material (knapp 1 lfd. Regalmeter) dem Herder-Institut übergeben. ${ }^{6}$ Ein besonderer Glücksfall war es, dass dann 2006 weiteres Material zur Familie, das in einem in Deutschland verbliebenen Zweig bewahrt worden war (v. Liphart in Marburg) der Dokumentesammlung des HerderInstituts übergeben wurde. ${ }^{7}$ Bei dieser zweiten Erwerbung handelt es

5 Vgl. das Projekt des Estnischen Wissenschaftsfonds (ETF Nr. 7554): „Ratshof und die Kunstsammlungen Liphart" am Kunstmuseum der Universität Tartu. Publiziert in: Unistuste Raadi. Liphartite kunstikogu Eestis = The Liphart family and their art collection in Estonia.

6 Vgl. Herder-aktuell, 10 (Januar-Juni, 2000), 16

Teile eines Familienarchivs kommen wieder zusammen", Archivnachrichten aus Hessen, 6/2 (2006), 29f. sich um einen vom Umfang her sogar etwas größeren Teil (ca. 1,2 lfd. Regalmeter).

Beide Teile des Familienarchivs zeugen von dem, was auf Grund der dramatischen Ereignisse des 20. Jahrhunderts eher zufällig erhalten ist, doch haben sie ähnlichen Charakter: Beide haben einerseits ihren Kern in den Unterlagen betr. Ratshof und die anderen Majoratsgüter und somit Materialien von hoher agrar-, wirtschafts- und sozialgeschichtlicher Bedeutung. Andererseits umfasst das Familienarchiv Nachlässe bzw. nachlassähnliche Materialien verschiedener Personen aus der Familie v. Liphart, die für die Kulturgeschichte der baltischen Länder im 19. Jahrhundert wertvoll sind.

\section{DIE FAMILIE V. LIPHART UND RATSHOF}

1751 kaufte Karl (oder auch Carl) v. Liphart (1719-1792) das Gut Ratshof in der Nähe von Dorpat und legte damit den Grundstein für die erfolgreiche Entwicklung seiner Familie zu einer der vermögendsten Großgrundbesitzerfamilien in Livland.

Der recht profane Name "Ratshof" erklärt sich aus der Tatsache, dass dieses Gut im Mittelalter dem Rat der Stadt Dorpat gehörte. ${ }^{8}$ Einige Unterlagen zur Verwaltung dieses Hauses, besonders aber auch der Stadthäuser in Dorpat sind in den Unterlagen in der DSHI vorhanden. Doch wird Ratshof immer mit dem Zusatz „Musenhof ${ }^{\prime \prime}$ verbunden sein „Das sehr reich ausgestattete Herrenhaus im Stil der Renaissance /---/ barg die größte private Kunstsammlung des Baltikums." ${ }^{10}$ Und: „Unsere Universitätsstadt Dorpat genießt den Vorzug, in ihrer nächsten Nähe eine Kunstsammlung zu haben, wie sich in den Ostseeprovinzen und weit darüber hinaus keine ähnliche findet. Die berühmte Galerie Liphart in Rathshof, von einheimischen und ausländischen Kunstfreunden und Forschern häufig besucht und uneingeschränkt bewundert, genießt auch im Ausland eines bedeutenden Rufs", so Alfred Grass in den Heimatstimmen von $1906 .^{11}$

8 Baltisches Historisches Ortslexikon, Teil I, hrsg. von Heinz von zur Mühlen, bearb. von Getrud Westermann (Köln, Wien: Böhlau Verlag, 1985), 488.

9 Thomson, Rauch, Schloß Ratshof in Estland: vom Musenhof zum Nationalmuseum, vgl. Anm. 2. 10 Schlösser und Herrensitze im Baltikum, hrsg. von Erik Thomson, Georg Baron von ManteuffelSzoege (Frankfurt am Main: Weidlich, 1963), 56.

Alfed Grast Schloß Ratshof und die Galerie Liphart", Heimatstimmen. Ein baltisch Jahrbuch, hrsg. von Carl Hunnius, Victor Wittrock (Reval: F. Kluge, 1906), 100. 
Diese Zitate stammen aus einer Zeit, als es Ratshof ${ }^{12}$ in seiner Blüte als Musenhof noch gab, also zur Zeit des letzten Majoratsherrn Reinhold Karl v. Liphart (1864-1940). 1919 wurde Ratshof im Zuge der estnischen Agrargesetzgebung enteignet. 1920 siedelte Reinhold v. Liphart nach Deutschland über und ging später nach Italien. Das in der Dokumentesammlung in Marburg vorhandene Archivgut enthält Informationen über diesen Zeitraum, insbesondere betr. die Dinge, die Reinhold v. Liphart dem Estnischen Staat überlassen musste: die Gebäude und Inventare, einige Teile der Kunstsammlungen (mehrere Werke konnte er aber auch mitnehmen). ${ }^{13}$ Die meisten Unterlagen über die weitere Geschichte von Ratshof befinden sich im Estnischen Historischen Staatsarchiv (Eesti Ajalooarhiiv) in Dorpat. Reinhold v. Liphart, eigentlich Arzt, schrieb nach dem Ersten Weltkrieg von Italien aus einige kunsthistorische Abhandlungen und konnte dabei die Tradition seiner Familie als Kunstsachverständige auch im 20. Jahrhundert lebendig erhalten. Zusammenhängende Unterlagen zur Familie beginnen in der Zeit, in der Ratshof erworben wurde. Zentrales Dokument ist hier eine „Nobilitierungsurkunde”: Der schwedische König Karl XI. erhebt seinen Offizier Friedrich Liphart am 16. März 1688 in den Adelsstand. Damit beginnt die livländische Geschichte dieses wohl aus Westfalen ${ }^{14}$ stammenden Geschlechtes. Zu den Gütern der Familie gehörten mit jeweils unterschiedlichen Besitzern ${ }^{15}$ neben Ratshof auch Neuhausen, Terrastfer, Marrama, Torma bei Laisholm, Toikfer ebenso wie Kondo bei Laisholm.

Insgesamt befinden sich bemerkenswerte Gutsakten zu Ratshof und zu den anderen Majoratsgütern in den Beständen des Familienarchivs Wegen der bruchstückhaften Überlieferung sind für die einzelnen Güter keine zusammenhängenden Akten vorhanden, also keine „Briefladen”,

12 Das Gutshaus Ratshof wurde Ende des 18. Jahrhunderts erbaut und hat als „Schloß” seine eigene Architekturgeschichte, die im Beitrag nicht näher beleuchtet werden kann, vgl. dazu u. a.: Ants Hein Eesti moisaarhitektuur - historismist juugendini $=$ Gutsarchitektur in Estland - vom Historismu bis zum Jugendstil, (Tallinn: Hattorpe, 2003), 38-39 und 83-86; Juhan Maiste, Eestimaa mõisad (Tallinn: Kunst, 1996), 11-119; Schlosser und Herrensitze im Baltikum, 56; Heinz Pirang, Das balt sche Herrenhaus, Band III (Riga. Jonck \& Poliewsky, 1930), 63.

(2)

4 Vgl. Thomson, Rauch, Schloß Ratshof in Estland: vom Musenhof zum Nationalmuseum (wie Anm. 2), 9, aber auch evtl. Sachsen als Herkunftsland in: Genealogisches Handbuch der Baltischen Ritterschaften Livland, Band 2 (Görlitz: Starke, [1943?]), 674.

5 Vgl. eine Ubersicht in: Zur Geschichte der Ritterschaften von Livland und Oesel, hrsg. von der Livl. Ritterschaft und der Oeselschen Ritterschaft (Pfaffenhofen/Ilm: Ilmgau Verlag W. Ludwig 1974), 168 sowie $195-199$. jedoch durchaus belangvolle Unterlagen für die Zeit, als die Familie v. Liphart diese Güter bewirtschaftete. Älteste Zeugnisse gibt es hier aus dem 18. Jahrhundert, z.B. eine „Haacken Liste derer Publiquen und Privaten Güther benebst derer Pastorathen, Rigischen und Wendenschen Creyses" von $1751^{16}$. Urkunden im Original wie in Abschriften bezeugen Rechtsakte vom 16. bis 18. Jahrhundert. Hierher gehören auch Korrespondenzen mit den jeweiligen Majoratsherren Liphart, aus denen wir einen anschaulichen sozialgeschichtlichen Einblick in die Majoratsführung erhalten. Aber nicht nur die Güter auf dem Lande, auch die anderen Besitzungen der Familie sind mit Akten vertreten. So finden wir innerhalb des Familienarchivs diverse Kaufkontrakte über Häuser etwa in der Stadt Dorpat. Auch die jährlich zu bezahlenden Quartierssteuern können wir aus den Quartiersbilleten Ende des 18. und Anfang des 19. Jahrhundert ersehen. ${ }^{17}$

\section{DIE FAMILIE V. LIPHART IM POLITISCHEN UND KULTURELLEN LEBEN}

Aus der gesellschaftlichen Stellung der Familie ergab sich selbstverständlich die Teilnahme am politischen Leben Livlands. Da waren zuerst einmal Sitz und Stimme auf den Landtagen. Darüber hinaus zeigten Angehörige der Familie aber auch ein weitergehendes Engagement in der Politik Livlands, der Ostseeprovinzen überhaupt und des Russischen Reichs. Davon zeugen Unterlagen über folgende Personen, die öffentliche Ämter bekleideten: der Garderittmeister Carl v. Liphart (1719-1792); der Hofrat und Landrat Reinhold Wilhelm v. Liphart (1750-1829), der Landmarschall Carl Gotthard v. Liphart (1778-1853). Reinhold Wilhelm v. Liphart war von 1807 bis 1828 ebenso Präsident der Gemeinnützigen Sozietät ${ }^{18}$, ein Amt, das viel Ansehen genoss. Dieses Amt bekleidete auch sein Enkel Karl Eduard v. Liphart (1808-1891) von 1847 bis 1862.

16 DSHI 110 Liphart I, 12.

iphart II, 03

18 Vgl. Hans Dieter von Engelhardt u. a., Die Livländische Gemeinnützige und Ökonomische Sozietät (1792-1939). Ein Beitrag zur Agrargeschichte des Ostseeraums (Quellen und Studien zu Baltischen Geschichte), 5 (Köln u. a.: Böhlau Köln, 1983) und Hubertus Neuschäfter, „Die Livländische Gemeinnützige und Okonomische Sozietät und ihr Verhältnis zur bäuerlichen Bevölkerung Livland in der zweiten Halfte des 19. Jahrhunderts", Bevolkerungsverschiebungen und sozialer Wandel in den Nordostdeutsches Kulturwerk, 1995), 127-140. 
In einer Art Vermächtnis schreibt Karl Eduard wohl an seinen Bruder Gotthard: „Da der Besitzer des Familien-Fideicommisses eine Stellung in der bürgerlichen Gesellschaft einnimmt, die ihm ausser den Pflichten eines Groß-Grundbesitzers noch diejenige auflegt[,] anregend, fördernd auf das geistige Leben seiner Mitbürger einzuwirken[,... ]freut es mich ihm zu diesem Zweck zwei wichtige Besitzthümer hinterlassen zu können, welche ihm hiezu nütze Mittel geben." ${ }^{19}$

Der estnische Historiker Tiit Rosenberg verbindet seine dreiteilige Abhandlung über die Familie v. Liphart mit dem eindringlichen Rat, das Bild über die die „Reitpeitsche schwingenden Barone” (hier in verkürzter Weise wiedergegeben) zu revidieren. Von dieser Vorstellung müsse man Abschied nehmen zugunsten einer differenzierteren Sicht, welche die Vielfalt unterschiedlicher ,Typen' unter den Gutsherren berücksichtigt. Dabei spielen die Beziehungen zu den estnischen und lettischen Bauern eine wichtige Rolle, die er ausführlich in drei Artikeln bearbeitet, und die gleichzeitig eine chronologische Übersicht über die jeweiligen Guts- bzw. Majoratsherren Liphart ergeben. Eine Familie, die sich ihren Reichtum hart erarbeitete und ihre Aufgaben innerhalb des Adels nicht nur als Gutsherren und Landespolitiker sah, sei ausdrücklich auch in einer weiteren Sicht zu beurteilen: ${ }^{20}$ In ihrem Reichtum sah diese Familie nämlich die Möglichkeit, Kunstschätze zu erwerben, an ihnen $\mathrm{zu}$ forschen oder forschen $\mathrm{zu}$ lassen und ihr bekanntestes Gutshaus, den Ratshof bei Dorpat, zu einem Ausgangspunkt vieler kunstfördernder Unternehmungen zu machen. Bis zur Etablierung staatlicher Kunstsammlungen und öffentlicher Kulturföderung hatten im 18. und 19. Jahrhundert vor allem private Sammlungen hervorragende kulturgeschichtliche Bedeutung. Die Familie v. Liphart hat aber auch in einer weiteren Sache nachhaltig gewirkt: Sie unterstützte einzelne Künstler und ihre Familien und förderte damit die Kultur Livlands ganz allgemein. Die erhaltenen Archivquellen ermöglichen dazu zahlreiche Beobachtungen.

Detaillierte Unterlagen zu Ratshof und zu der Übernahme von Ratshof und seinen Kulturgütern durch den Estnischen Staat 1920, befinden sich, wie schon gesagt, im Historischen Staatsarchv Estlands. In der DSHI in

19 DSHI 110 Liphart II, 1-16, 37. Das Dokument stammt aus den 1880er Jahren und ist wohl als Entwurf anzusehen.

Rosenberg „Liphartid Liivimaa mõisnikuna I = Die Lipharts als Gutsbesitzer Livlands”, 12 (wie Anm. 4).
Marburg ist eine Liste überliefert: „,v. Liphart'i asjad, mis võetud linna korteris” („Dinge der v. Lipharts, die aus der Stadtwohnung genommen wurden"), die sich auf die Kunstschätze im Stadtpalais der Familie in Dorpat bezieht. Über die 1920 aus Estland ausgeführten Kunstschätze geben die vom letzten Majoratsherrn Reinhold v. Liphart veranstalteten Kunstauktionen bzw. deren Kataloge Aufschluss, die Vielfältigkeit und Größe der Sammlungen erahnen lassen. ${ }^{21}$

Die Familie v. Liphart hat nicht nur zahlreiche Künstler gefördert, aus ihr sind auch selbst Künstler hervorgegangen: Der bekannteste ist wohl Ernst Friedrich (1847-1932), dann aber auch Carl Eduard (1900-1976) oder Reinhold Karl (Carl) (1864-1940). Von den beiden erstgenannten gibt es keine Zeugnisse in den Materialien der DSHI. ${ }^{22}$ Unterlagen von oder über Reinhold v. Liphart wurden im obigen Abschnitt über die Gutsakten schon erwähnt.

Weniger als Künstler denn als Kunstsammler und -sachverständiger und als Mäzen ist Karl Eduard v. Liphart (1808-1891) berühmt geworden. ${ }^{23}$ Seine Bedeutung für Ratshof und die im Baltikum einmalige Sammlung von Kunstschätzen ist unbestritten: In Estland wird er in Enzyklopädien meist als einziger Vertreter der Familie genannt ${ }^{24}$. Die weitreichende Bedeutung Karl Eduard v. Lipharts wird vielleicht auch darin deutlich, dass sein Sohn Ernst Friedrich, der in Kunstkreisen sicher bekanntere Künstler, meist nur ,als Sohn des bekannten Kunstforschers

21 Vgl. DSHI 110 Liphart II, 7-1: Kopenhagen 1920, Amsterdam 1921, München 1931. 22 Vgl. jedoch: Lexikon deutschbaltischer bildender Künstler. 20. Jahrhundert, hrsg. von Kuno Hagen unter Mitarbeit von Margarete Hagen (Köln: Verlag Wissenschaft u. Politik, 1983) [Carl Eduard v. Liphart; Ernst Friedrich v. Liphart und Reinhold Carl/Karl v. Liphart]; Malerwerke des neunzehnten 1941), bzw. 1891-1901 [Ernst Friedrich v Liphart]; Lexikzon baltischer Künstler, Pantheon Verlag 1941), bzw. 1891-1901 [Ernst Friedrich v. Liphart]; Lexikon baltischer Kunstler, hrsg. von Wilhelm Neumann (Riga: Jonck \& Poliewsky, 908 ) [Ernst Friedrich v. Liphart]; Baltische Maler und Bildhaue des XIX. Jahrhunderts. Biographische Skizzen mit den Bildnissen der Kunstler und Reproduktionen nach ihren Werken, bearb. von Wilhelm Neumann (Riga: A. Grosset, 1902) [Ernst Friedrich v. Liphart]. 23 Zu Karl Eduard v. Liphart und der Familie v. L.: vgl. u. a. Genealogisches Handbuch der baltischen Ritterschaften, herausgegeben von den Verbänden des livländischen, estlandischen und kurländischen Stammadels, Teil: Livland, Band II (Schlesien: Görlitz, 1929), 679-685; Thomson, Rauch, Schloß Ratshof in Estland: vom Musenhof zum Nationalmuseum; Juta Keevallik, „Karl Eduard von Liphart”, Jahrbuch des baltischen Deutschtums, 39 (1992), 38-41; Rosenberg (wie Anm. 4); Neumann, Lexikon baltischer Kunstler, 127; Adolf Friedrich von Schack, ,Karl Eduard von Liphare, Baltische Monatsschrift, 41 (Reval 1894), 424-432, In Fid Sahk, "Geteilte Freude und gelehrsame Abende. Uber die Graphiksammlung und die künstlerischen Abende im Haus des Kunstkenners Karl Eduard von Liphart", Baltic Journa of Art History, 6 (2011), 303-328.

"Estnische Lebensgeschichten", Eesti Entsüklopeedia 14 (Tallinn: Eesti Entsüklopeediakirjastus, 2000), 250. 
Karl Eduard v. Liphart"25 bezeichnet wird. Auch wenn Karl Eduard v. Liphart kaum öffentlich im künstlerischen und kunsthistorischen Leben Livlands erscheint, so hat er doch mit seinem Kunstverstand und seiner Fürsorge für Kunst und Kultur seine Zeit entscheidend geprägt.

\section{DIE BEDEUTUNG DER KORRESPONDENZEN UND ANDERER SELBSTZEUGNISSE}

Gerade bei solch hervorragenden Menschen ist das, was in persönlichen Äußerungen gesagt oder geschrieben wird, auch von allgemeiner historischer Bedeutung. In dem hier vorgestellten Archivbestand sind einige bedeutende Skripte und Korrespondenzen von Karl Eduard v. Liphart der Forschung zugänglich.

Neben den rein geschäftlichen Briefen, die jedoch stets einen sehr persönlichen, manchmal sogar familiär-freundschaftlichen Stil haben ${ }^{26}$, sind hier die kunsthistorischen Notizen und Notizbücher ${ }^{27} \mathrm{zu}$ nennen, in denen immer wieder Zeichnungen Karl Eduards vorkommen. In seinen Korrespondenzen mit Kunsthäusern in vielen Ländern lässt Karl Eduard v. Liphart keinen Zweifel an seinem Kunstverstand, aber auch nicht an seiner Begeisterung für „,schöne Werke”; für Kunsthistoriker aufschlussreiche „Blätter” und andere Notizen und Aufzeichnungen ${ }^{28}$ enthalten Äußerungen wie: „,Die Mère de Rembrandt hätte mir angestanden /---/ Sein Portrait № 20 mit der Mütze und der einen Feder würde ich auch ganz gern haben. Den Both № 1 /---/ mit weißer Figur hätte ich auch gar gern, nur freilich zu raisonablem Preise /---/."29

Die in der DSHI überlieferte Korrespondenz Karl Eduard v. Lipharts weist jedoch noch viel weitergehende Aspekte als die des Kunstsachverständigen auf. Im Familienarchiv existieren auch zahlreiche Briefe aus dem engeren Familienkreis. Dieser für die historische Forschung über Livland nicht hoch genug zu schätzende Bestand wird ergänzt durch einen weiteren Teil, nämlich den der freundschaftlichen Korrespondenz. An dieser Stelle müssen einige wenige Hinweise genü-

25 So u. a. bei Neumann, Lexikon baltischer Künstler.

26 Vgl. DSHI 110 Liphart II, 2-17: das sind Briefe Karl Eduard von Lipharts an den Generalbevollmächtigten (Th. Saag-Wulffius, Hofgerichts-Advokat Dorpat) des Liphart'schen Majorats $880-1890$

27 Als Beispiele: DSHI 110 Liphart I, 2; DSHI 110 Liphart II:1-16; II: 2-6

29 Notizen an Weber und Kolk in Köln 1847, DSHI 110 Liphart I, 2,6. gen: $\mathrm{Zu}$ beginnen ist mit Briefen aus der Studienzeit. Aus archivischer Sicht ist es erfreulich und für die weitere Forschung überaus nützlich, wenn zusammengehörende Teile von Korrespondenzen, die durch das Schicksal des 20. Jahrhunderts getrennt waren, sich nun in einem Archiv wiederfinden: Dazu gehört etwa der Briefwechsel zwischen Liphart und Paul Stoppelberg (1809-1837) ${ }^{30}$, einem Kommilitonen Lipharts im Medizinstudium an der Universität Dorpat. Im Familienarchiv Liphart Teil I befindet sich ein Konvolut mit Briefen an Paul Stoppelberg, der schon früh verstarb, so dass eventuell aus diesem Grund das Bündel von Briefen Lipharts an deren Verfasser zurückgeschickt wurde und sich heute entgegen dem archivischen Prinzip auch in Marburg befindet. Im Familienarchiv Teil II gibt es mehrere Briefe von Stoppelberg an Liphart aus den 1830er Jahren. ${ }^{31}$

Aus der gleichen Zeit, also aus den 1820er und 1830er Jahren, finden sich Briefe von Studienkollegen, Medizinern, aber auch Kunsthistorikern: So fallen Namen wie der des Wolmarer Stadtarztes Johann Erdmann (1809-1858), auch Professor für Medizin in Dorpat, oder aber des bekannten und in diesem Band im Vordergrund stehenden Zeichners Woldemar Krüger (1808-1894) ${ }^{32}$, der schon als Kind nach Ratshof kam und mit Karl Eduard v. Liphart gemeinsam erzogen wurde und den eine lebenslange Freundschaft mit den Lipharts verband. Um die Vielfältigkeit der Korrespondenz zu veranschaulichen, seien hier noch zwei spätere Kontakte Lipharts genannt: Von 1875 bis 1880 schreibt Franz Lenbach (später von Lenbach, 1836-1904) aus München an den „hochverehrten und lieben Freund", genauso Ignaz Maria von Olfers (1793-1872) in seiner Eigenschaft als Generaldirektor der Königlichen Museen in Berlin. Durch familiäre Beziehungen gibt es auch ein umfangreiches Konvolut mit Briefen des berühmten Geigers Ferdinand David (1810-1871) an seinen Schwager Karl Eduard v. Liphart.

Für die im Familienarchiv Liphart überlieferten Briefe ist, wie oben schon gesagt wurde, ein außergewöhnlich freundschaftlicher Ton charakteristisch. Dieser Umstand sollte nicht unerwähnt bleiben, denn er gilt für jene Kontakte, die die Familie v. Liphart zu vielen Personen des öffentlichen Lebens allein schon wegen ihrer politischen Stellung

30 Album Academicum der Kaiserlichen Universität Dorpat, bearb. von Arnold Hasselblatt, Gustav Otto (Dorpat: Verlag von C. Mattiesen, 1889), Matrikelnr. 2463.

32 Neumann, Lexikon baltischer Künstler, 100 
selbstverständlich unterhielt; und er gilt besonders für jene anderen Verbindungen, die uns heute vor allem aus kulturgeschichtlicher Sicht besonders interessieren. Jede Untersuchung, die sich mit personen- und sozialgeschichtlichen Aspekten beschäftigt und die herausfinden will, mit welchen Personen des politischen, wirtschaftlichen und kulturellen Lebens die Familie v. Liphart Kontakt hatte, wird sich natürlich immer sehr eng an die einzelnen Angehörigen der Familie halten und deren Bedeutung für die baltische, deutsche, russische und für die gesamteuropäische Kulturgeschichte berücksichtigen müssen.

Auch das Mäzenatentum der Familie v. Liphart tritt gerade in den Korrespondenzen überaus deutlich zutage. Daraus geht auch klar hervor, dass die Angehörigen der Familie dies als ihre sich aus der wirtschaftlichen und kulturellen Stellung ergebende besondere Verpflichtung empfanden. Viele Briefe an die Familie beginnen mit einem Dank für die großzügige Unterstützung, so auch die in diesem BJAH publizierte Briefe des Künstlers Woldemar Krüger.

Die Facetten Liphart'schen Mäzenatentums sind vielfältig. Zu erwähnen ist der Geist der Freiheit, Dinge beim Namen nennen zu dürfen, auch wenn damit der allseits geschätzte Mäzen kritisiert wurde: Neben vielen anderen hatte auch der berühmte Arzt und Schriftsteller Georg Julius von Schultz-Bertram (1808-1875) engen Kontakt zu seinem Studienfreund Karl Eduard v. Liphart. Schultz-Bertram gibt in ungewöhnlicher Offenheit eine sehr kritische Sicht auf das adlige Leben im Schloss Ratshof zu erkennen, eine Offenheit, die sicherlich nur aus einer wirklichen Freundschaft, getragen von großem gegenseitigen Vertrauen und Respekt, resultieren konnte. ${ }^{33}$

$$
\text { SCHLUSS }
$$

Was in den Korrespondenzen immer wieder mit Bezug auf die Familie v. Liphart angesprochen wurde, gilt auch für den ganzen Musenhof Ratshof, so wie es Alfred Grass 1906 zum Ausdruck brachte: „/----/ so ist es in der Tat eine imposante Fülle edelsten Kunstbesitzes, den die schö-

33 Vgl. Dorothee M. Goeze: „„,Ich möchte meine Feder meinem lieben baltischen Vaterlande weihen". Aus Briefen Georg Julius von Schultz-Bertrams an Karl Eduard von Liphart", Buch und Bildung im Baltikum. Festschrift fur Paul Kaegbein zum 80. Geburtstag, (Schriften der Baltischen Historischen LIT, 2005), 457-466. nen Räume des Rathshoffschen Schlosses bergen, eine Fülle, die nur in dem glücklichen Umstand ihre Erklärung findet, dass der Kunstsinn mehrerer Generationen des Hauses sein Streben auf immer größere Erweiterung dieses Besitzes gerichtet hat, auf den die Majoratsherrn von Rathshof ein Recht haben stolz zu sein." 34

Dass die Majoratsherren von Ratshof und weitere Angehörige der Familie v. Liphart auf diesen Kunstbesitz stolz waren, ihn aber auch in einem weiten Kontext als Aufgabe, als Verpflichtung verstanden und wahrnahmen, um Kunst und Kultur und deren Erforschung in Livland zu befördern, lässt sich selbst aus den in Marburg erhaltenen, eigentlich geringen Resten des Familienarchivs deutlich belegen. Die umfassende Auswertung dieses Familienarchivs steht erst am Anfang. Sie verspricht schon jetzt zahlreiche neue Erkenntnisse.
34 Vgl. Grass, „Schloß Ratshof und die Galerie Liphart”, 101, daneben erhalten wir aber auch Informationen über "Schloss Rathshof und seine Kunstschätze” in einem Artikel von Carl Krüger um 1900 in der Liphart II, 4-4. 
Dorothee M. Goeze: The von Liphart Family Through Their Archive in The Herder Institute in Marburg Keywords: Liphart Family Archive; Herder Institute; Document Collection; Von Liphart; Baltic Study

\section{SUMMARY:}

The article introduces the archive of the von Lipharts, a noble Baltic German family, stored in the document collection of the Herder Institute in Marburg (DSHI). The von Lipharts and their Raadi Manor near Tartu were of great importance to Baltic cultural history, and the archive reflects their role in the politics, economy and cultural life of Livonia, as well as the family's subsequent status. A more in-depth presentation is made of the various materials in the archive that are related to the communications and activities of the Liphart family related to culture and art. In addition to describing the development and character of the family archive at the Herder Institute, the article also provides a survey of the historiography of the Liphart family and the latest research based on the collections in the archive.

\section{CV:}

Dorothee M. Goeze works in the document collection at the Herder Institute She has studied comparative linguistics and history at the University of Mainz. Her field of research is the languages, history and cultural history, as well as archival science, of the Baltic countries, especially Estonian language and history. Dorothee M. Goeze is a member of the Learned Estonian Society and the Baltic Historical Commission. She has written numerous articles dealing with the materials in the document collection of the Herder Institute and Baltic (cultural) history for both German and Estonian publications. 